\title{
Intelligent Computer-Aided Prostate Cancer Diagnosis Systems: State-of-the-Art and Future Directions
}

\author{
Rachid Sammouda ${ }^{D},{ }^{1}$ Abdu Gumaei ${ }^{(D},{ }^{1}$ and Ali El-Zaart ${ }^{2}$ \\ ${ }^{1}$ Department of Computer Science, College of Computer and Information Sciences, King Saud University, Riyadh, Saudi Arabia \\ ${ }^{2}$ Department of Mathematics and Computer Science, Faculty of Sciences, Beirut Arab University, Beirut, Lebanon
}

Correspondence should be addressed to Rachid Sammouda; rsammouda@ksu.edu.sa and Abdu Gumaei; abdugumaei@gmail.com

Received 15 March 2021; Accepted 1 June 2021; Published 7 July 2021

Academic Editor: G. Muhiuddin

Copyright ( 2021 Rachid Sammouda et al. This is an open access article distributed under the Creative Commons Attribution License, which permits unrestricted use, distribution, and reproduction in any medium, provided the original work is properly cited.

\begin{abstract}
Prostate Cancer (PCa) is one of the common cancers among men in the world. About $16.67 \%$ of men will be affected by PCa in their life. Due to the integration of magnetic resonance imaging in the current clinical procedure for detecting prostate cancer and the apparent success of imaging techniques in the estimation of PCa volume in the gland, we provide a more detailed review of methodologies that use specific parameters for prostate tissue representation. After collecting over 200 researches on image-based systems for diagnosing prostate cancer, in this paper, we provide a detailed review of existing computer-aided diagnosis (CAD) methods and approaches to identify prostate cancer from images generated using Near-Infrared (NIR), Mid-Infrared (MIR), and Magnetic Resonance Imaging (MRI) techniques. Furthermore, we introduce two research methodologies to build intelligent CAD systems. The first methodology applies a fuzzy integral method to maintain the diversity and capacity of different classifiers aggregation to detect PCa tumor from NIR and MIR images. The second methodology investigates a typical workflow for developing an automated prostate cancer diagnosis using MRI images. Essentially, CAD development remains a helpful tool of radiology for diagnosing prostate cancer disease. Nonetheless, a complete implementation of effective and intelligent methods is still required for the PCa-diagnostic system. While some CAD applications work well, some limitations need to be solved for automated clinical PCa diagnostic. It is anticipated that more advances should be made in computational image analysis and computer-assisted approaches to satisfy clinical needs shortly in the coming years.
\end{abstract}

\section{Introduction}

Cancer is one of the most critical health issues globally, in terms of morbidity, mortality, and its social, economic, or quality of life, affecting one in three people throughout their lives [1]. For all age groups, cancer became the leading cause of death worldwide for men and women after cardiovascular diseases [2]. Prostate cancer is the most frequent tumor location in men (excluding nonmelanoma skin tumors) and the third leading cause of death from cancer, in both cases behind cancer of the lung and colorectal. It is estimated that one in six men will develop prostate cancer in their lifetime [3]. The probability of developing prostate cancer increases with age so that nine out of ten cases appear in people over 65 years of age [4].

Diagnostic practices and therapeutic options have continued to evolve for detecting prostate cancer. Precisely, recent advances in prostate imaging make it possible to detect tiny tumors and guide treatment [5]. ComputerAided Diagnosis (CAD) software and tools are designed to aid physicians in diagnosing suspicious areas of the image. The principle is to extract from one or more types of medical images (such as MRI, CT, US, and PET) discriminating characteristics of the pathology than to develop a prediction model from a database learning. This empirical model then makes it possible to quantify the probability that an area of interest or a voxel of a test image is pathological [6].

This work's main contributions and goals are to conduct a detailed review of existing CAD methods and approaches for identifying prostate cancer from images. Additionally, we proposed two research methodologies of CAD systems based on Near-Infrared (NIR), Mid-Infrared (MIR), and Magnetic Resonance Imaging (MRI) techniques. 
The rest of the paper is organized as follows. Section 2 provides detailed background on prostate cancer. Section 3 describes the near-infrared (NIR) and mid-infrared (MIR) images. Section 4 describes the two research methodologies of a CAD system on NIR, MIR, and MRI images. Section 5 gives a detailed explanation of the clinical application. In Section 6, we discuss our observations and list the future directions. Finally, we conclude our work in Section 7.

\section{Background}

The prostate is one of the essential glands in humans that is known as a walnut-sized gland [1]. The anatomical structure of the prostate gland is divided into three standard zones. The peripheral zone (PZ) is the most significant part of the gland that accounts for $70 \%$ of a gland volume and extends from the base to the apex of the prostate gland. The second part is the central zone (CZ), representing $25 \%$ of the gland, and the transition zone (TZ) represents the $5 \%$ rest of the prostate. The cancer of the prostate is commonly diagnosed at the $\mathrm{PZ}$, where around $70 \%$ arises. On the other hand, $10 \%$ to $20 \%$ of prostate cancer cases are affected by a tumor in the TZ. About $5-10 \%$ prostate cancer is located in CZ [2].

Since the late 1980s and 1990s, there is a revolutionary shift in the health care agenda and diagnosis techniques of the developed world. Cancer starts taking place in the leading causes of death in humans. In the report [3], cancer named to be the second cause of death after heart diseases. Within different forms of cancer, the most prevalent source of male cancer and the second leading cause of cancer death after lung cancer is Prostate Cancer (PCa). Among men, prostate cancer is a globally common cancer. It grows rapidly [4]. Therefore, prostate cancer and its diagnosis techniques make a priority to prostate cancer research. According to the American Cancer Society Report of 2019, prostate cancer cases in the US up to 174,650 patients will be diagnosed, and the estimated number of deaths will reach 31,620 cases. Current studies have predicted that the risks of cancerous disease in Asia will be rising in the future, especially in the growth countries [5]. The paper [6] anticipated that cancer would significantly increase on the continent of Asia, where $60 \%$ of the global citizens live. Therefore, macro safety strategies in the future are a significant need for dealing with cancer. Early diagnosis of prostate cancer plays an essential role in survival rate or chronic conditions.

The case of a population screening process has been a topic of continuous discussion since the early 1990s [7]. PCa is one of the leading cancers causing death through the world [8]. Due to the limitations in the existing clinical practices, accurate and easy diagnosis and assessment of PCa still needed improvements. Computer-aided diagnostic approaches would probably play an influential role in addressing this problem in the near future [9]. Nonetheless, since the early nineties, the need for community screening had been the focus of ongoing debate [7]. In medical tests for prostate cancer, the immunoassay-based assessment of blood levels of Prostate Specific Antigen (PSA) is generally accepted as the first phase in clinical screening for prostate cancer. Virtual (DRE) and transrectal ultrasounds (TRUS) are usually taken for patients with a higher PSA level.

Many large-scale prostate types of research focused on specific antigen studies, and they have shown reduced death risk related to PCa [10-12]. Unfortunately, as these approaches are simplistic and lack precision, assessing the occurrence and diagnosis of the disease is now carried out exclusively through a histopathological review of biopsy samples collected through TRUS, or MRI guidelines. However, some major studies have reported contradictory results, suggesting that PSA screening has no critical impact on PCa death rates $[13,14]$. Due to the less importance of the PSA in the diagnosis process, it has been issued or marked with the " $D$ " letter symbol by the United States Preventive Services Task Force (USPSTF). It culminated in a shift away from the use in the United States [15]. In detecting clinically significant tumors, the random biopsy approach is also vulnerable to low sensitivity [16-19]. Although TRUS is more reliable and cost-effective than mp-MR, its low sensitivity does not render a broad patient population [20]. Through this restriction, the imaging of endorectal MR leads to the PSA, DRE, and biopsy outcomes despite localization and measurement of cancer scale and extension [21, 22].

For endorectal images, the ROC curve for locating cancer is higher for apex, center, and base than for DRE. Additionally, endorectal MR imaging is more reliable in ingrowth and prostate early diagnosis, especially for the prostate base against TRUS controlled biopsy in the localization of the tumor [23]. This paper emphasis the focus of Magnetic Image Resonance (MR) Images more than transrectal ultrasound (TRUS) because of the success of MR in estimating the volume of PCa. The use of TRUS gives underestimate calculations. Therefore, the volume is used to calculate PSA density, and TRUS leads to inaccurate analysis. MR provides a more accurate estimation of prostate volume compared to TRUS [24]. Identifying the exact location of prostate cancer using transrectal ultrasound is inaccurate as more as MRI images. The recent prospective study presented that the sensitivity of the diagnosis process of a model that uses transrectal Ultrasound (TRUS) to diagnose $\mathrm{PCa}$ is only $70.4 \%$ [25].

Song et al. [26] used deep convolution neural networks to perform a classification task on 195 patients with localized PCa collected from a prostate database. The data were sequence of T2W, DWI, and ADC combinations. It was related to two classes of cancerous data divided to 215 for training and 23 for validation, and 23 for testing; the second class was healthy people, and it is divided into 229 training and 25 for validation, and 32 for testing. After implementing DCNNs, the outcome accuracy reached 94\%. Treviño et al. [27] examined rapid perceptual processing on T2W images, and the rapid radiological perception could be observed using T2W multislice imaging. The MR has the ability to extract the perceptual processing quickly and could also detect and localize lesions even in complex imaging modalities.

Chirra et al. [28] used about 147 patient T2-weighted MR datasets downloaded from four different sites containing 406 
three-dimensional voxel-wise radiomic features from five different families (gray, Haralick, gradient, Laws, and Gabor) and evaluated in a reproducible either tumor or nontumor and the discrimination process of tumor regions, and they reached an accuracy of $\approx 0.8$ in the classification phase. A recent review [29] concluded the independent behavior of the sensitivity in the diagnosis process of PCa on MR mages and many factors such as the enrollment of patient (consecutive or not consecutive), the strength of MR images (1.5 Tesla or 3 Tesla), the reference standard of bias risk (low risk of bias and high risk of bias), and whether readers are blinded or not to the histological findings (blind or not blinded).

2.1. Near-Infrared (NIR) and Mid-Infrared (MIR) Images. With the assistance of Maestro in vivo imaging system, imaging is conducted. The Near-Infrared (NIR) and MidInfrared (MIR) images are acquired by using the appropriate filter set (deep red filter set for Prostate Specific Membrane Antigen (PSMA)-1-IR800 and yellow filter set for PSMA-1Cy5.5). During this process, different points are chosen for imaging. The temperature of $37^{\circ} \mathrm{C}$ is tuned for the imaging bed through imaging operation. A nose cone is adjusted with an imaging bed for isoflurane inhalation.

Fluorescent molecular tomographic (FMT) images are obtained by using the FMT2500 device (Perkin-Elmer, Waltham, MA), and the three-dimensional reconstructions of fluorescent signals are captured using the accompanying software, TrueQuant. Quantification of fluorescent signals is also obtained by calibration of PSMA-1-IR800 and PSMA-1-Cy5.5 by the $780 \mathrm{~nm}$ and $680 \mathrm{~nm}$ channels, separately.

Several similar NIR/MIR images are collected by developing PSMA-targeted NIR/MIR optical imaging probes. These are used for analyzing and visualization of prostate cancer intraoperatively. A high affinity PSMA ligand (PSMA-1) is synthesized with low molecular weight and further labeled using commercially available NIR/MIR dyes: Cy5.5.4 and IRDy800 demonstrated the utility of such probes to selectively bind of the prostate tumor in targeting both orthotropic and heterotopic prostate tumors.

2.1.1. NIR and MIR Acquisition. Intuitively, image acquisition is the first stage of any vision system. In the research methodology of CAD system on NIR and MRI images, the tissue samples will first be normally evaluated by experienced pathologists to be separated into the following class labels.

(1) Stroma: STR (normal muscular tissue);

(2) Benign prostatic hyperplasia: BPH (a benign condition);

(3) Prostatic intraepithelial neoplasia: PIN (a precursor state for cancer);

(4) Prostatic carcinoma: PCa (abnormal tissue development corresponding to cancer).
Figure 1 demonstrates the NIR and MIR multispectral acquisition system. In this system, a liquid crystal tunable filter (LCTF) (VariSpec) is plugged in the optical path in the middle of the chilled charge-coupled device (CCD) camera and the light source. The LCTF has a bandwidth accuracy of $0.25 \mathrm{~nm}$ to $20 \mathrm{~nm}$. The wavelength is controllable over several spectrums. This allows capturing a different MIR multispectral images of the tissue samples at diverse spectral frequencies. Usually, the experiments are carried out on varying numbers of bands to illustrate the impact of multispectral imaging.

2.1.2. Challenges of Using NIR and MIR Images. One of the challenges of using these images is interpreting the imaging data to reflect cancer margin accurately. It is difficult to obtain accurate, reproducible, intent, and precise assessments in cancer research. The dilemmas occurred because of the biological dissimilarity, variability of personnel, and natural unpredictability. The NIR imaging technique characterizes cancer by adding fluorescent information to each pixel in the acquired image. For prostate cancer, the NIR imaging CAD system could be analyzed and classified to construct several sharp diagnostic rules.

2.2. Magnetic Resonance Imaging (MRI). The PCa representation in the past decade has changed substantially. Nevertheless, the mp-MR of prostate was mostly confirmed by the increases in magnet strength between 1 and 3 Tesla. The mp-MR is used for anatomic and functional imaging techniques: the anatomical imaging includes T1 or T2weighted stimuli, and the dynamic contrast-enhanced (DCE) imagery is included in functional imaging. An MRI of 1.5 Tesla is currently recommended with an endorectal coil or an MRI of 3 Tesla without using an endorectal coil. The MRI scan of multiples parameters is one type that produces accurate photographs of the prostate rather than a normal MRI scan, because it combines up to four different image types. From the mp-MR, the doctor can conclude whether or not cancer is present in the tested prostate and how rapidly cancer is expected to develop. The combination of different images of the MRI provides vast quantities of data. Supporting techniques or methods like CAD is vital for a fast, accurate, and reliable clinical decision. The PCa diagnoses are necessary to interpret MRI images for prostate radiologists, and this knowledge is not widely available. Intelligent CAD implementation will significantly increase the efficiency of less experienced PCa diagnosis observers.

$m p-M R$ Acquisition. In 2012, a discussion was raised up for the use of a combination of images (T2W, DWI, MRS, and DCE) and practical methods in the Prostate Imaging Reporting and Data System (PI-RADS) on prostate MR guidelines Barentsz et al. [30]. In comparison, each type of these images has specific points of strength that will help us in the diagnosis process. For the anatomical structure of prostate gland, T2W images are the ideal choice; DWI and MRS add the characterization of lesions, while DCE-MR imaging has a high sensitivity to cancer detection. In 


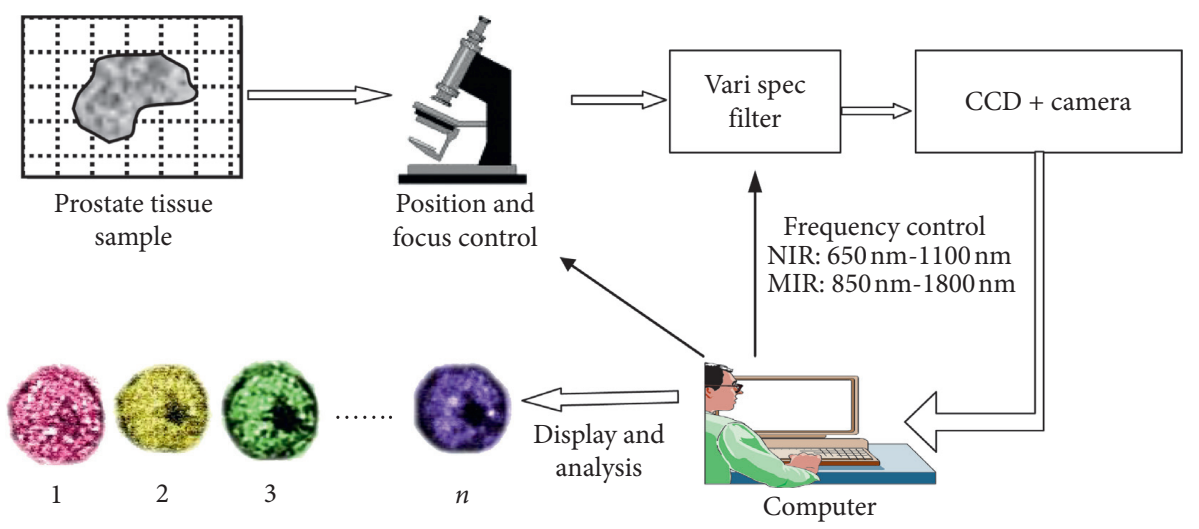

FIgURE 1: NIR and MIR acquisition system.

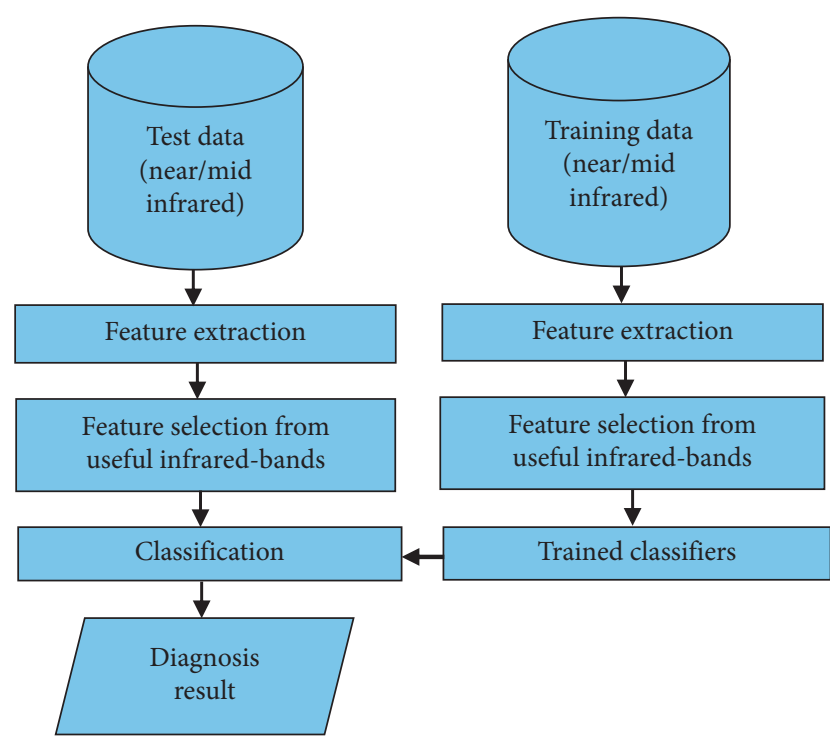

Figure 2: Flow chart of CAD system using NIR or MIR images.

PIRADs methodologies, T2W, DWI, and DCE are the elementary components used during the mp-MR test [31]. The DWI is the central sequence of determination for the PZ. For the $\mathrm{TZ}$, and the $\mathrm{T} 2 \mathrm{~W}$ is the primary sequence of judgment. A combination of anatomical and functional imaging is required in clinical practice to achieve high and reliable results. Recent studies have shown an increased interest in CAD systems development to detect PCa on the mp-MR approach and its representation [32-35]. In mp-MR CAD devices, T2W-MR images are commonly used. The power of multiparameter MR comes from gathering the strength points of T2W, DWI, and DCE-MR types. Therefore, this type is the most widely used currently.

T2WI and T2 Mapping. Taking the anatomical structure of the prostate with a T2W image is an effective way to obtain the ideal acquired image. The first and the most important step in an mp-MR protocol is the acquisition phase for a high-resolution $\mathrm{T} 2 \mathrm{~W}$ prostatic image. In $\mathrm{T} 2 \mathrm{~W}$ images, hyperintense signals are present in the peripheral zone $\mathrm{PZ}$ of the prostate, while a low signal exists in the central area and on TZ, which allows an easy and straightforward recognition for the defined zonal prostate anatomy. The Peripheral Zones of the PCa usually are represented as a low-signal region in T2W. Nevertheless, the pattern of tumor existence and growth will affect the appearance of the prostate anatomical structure. In [36, 37], images in T2W-MRI type for prostate were used as a specific technique for PCa detection in the transition zone. Due to the reliability of this type, it has been used for determining the area of interest of the tumor. At the same time, T2W-MR images contain more dark pixels than luminous pixels in the tumor area of interest, whereas the normal tissue ROI has more luminous pixels than dark pixels. A CAD system can use different characteristics, including fractal elements, textural features, and signal strength. As PCa and PZ usually have different textures in T2W-MR images [38] and since mp-MR can be challenged in the detection of TZ cancer [39], a CAD system can help identify irregular lesions that can examine feature-based positions of the lesion. The device can also be used to diagnose Transition Zone cancer. The quantitative T2 values are given by $\mathrm{T} 2$ maps images. Because of the requirements of traditional T2 mapping methods, such as excessive scanning times for multiple single eco-acquisitions with a variety of echo-time settings, the T2 mapping in most clinical applications is not supported. Several new sequences have recently been used to speed up T2 quantification [39-42]. The $\mathrm{T} 2$ values of the malignant tumor areas that had been histologically demonstrated were significantly lower than suspicious nonmalignant lesions or the standard areas [40]. Quantitative T2 assessment increases PCa detection accuracy and/or sensitivity [43], and the evaluation of aggression can be incorporated into CAD systems $[44,45]$.

DCE-MR Images. DCE image is an essential oncological tool for defining prostate tumors. Indicators referring to signal strength should be used; because the semiquantitative DCEMRI results are proportional only to the patient, and the baseline amplitude depends on the patient and the MRI imaging guidelines. DCE is so sensitive to the changes of vascular, blood flows, or extra-cellular space. So, it is practical to use quantitative or semiquantitative techniques to analyze and measures the changes of vascular, blood 
flows, or extra-cellular space. The clinical application of DCE-MRI in prostate cancer is differentiating between malignant lesions and healthy tissues by enhancement and contrast agent washing out. The Pharmacokinetic (PK) compartment model is one of the most commonly used quantitative analytical approaches of DCE-MRI imaging. PK allows the generation of the PK parameters, such as K-trans transferring gadolinium contrast from the vasculature to the tumor, which reflects forward vascular infusion and permeability of the contrast uptake and washout [46] and the methodology for estimating input functions, which can have significant effects on the PK model-ling parameters measured [47]. The experimental findings from a DCE-MRI acquisition can be replicated by an analytical approach based on phenomenological universities $[48,49]$. To analyze DCEMRI data, several CAD systems were developed. For example, Vos et al. [50] have developed a CAD model that can differentiate PCa tissues and nonmalignant Peripheral Zone tissues. Moreover, the system has achieved 83\% (0.75-0.92) as an average accuracy. An automated segmentation model has been developed to improve the accuracy of CAD diagnosis [51] to produce five-level scores for checked images in DCE-MRI and T1 images. There were many attempts to construct and design an automated prostate cancer diagnosis system. Puech et al. developed a prostate CAD system $[52,53]$. Typically, DCE-MRI requires enough acquisition time to produce a good resolution image.

In contrast, DCE-MR has lower spatial resolution than other sequences, mainly, if DCE-MR is carried out quickly over a short period. Limitations to DCE-MR interpretation involve duplication of enhancement features between benign and malignant TZ areas. There is also major hypervascularity in benign prostatic hyperplasia and other benign inflammatory disorders within the TZ [54]. Contrast-enhancement diagnostic models have reduced performance when used in zones, so zone-specific models should improve mp-MR PCa classification [55].

Diffusion-Weighted MR Images. In general, the DWI is generated with different b-values to measure the apparent diffusion coefficient (ADC), and the ADC is shown as ADC map for each pixel of the image. The spread of water molecules in tumor tissue reflects the architecture of tissues, such as cell density and the nucleus-to-cytoplasm ratio and $\mathrm{ADC}$ values. As a predictor of Gleason score in $\mathrm{PCa}[56,57]$ for these reasons, the ADC values have been given attention. Data from DWI trials have shown that tumor aggressiveness can be detected using type DWI [57-59].

Bi-Parametric MR Images. The drawbacks of DCE imaging, which are the expensive contrast agent management, long scanning period, have led to the use of new types of images. A biparametric MR (bp-MR) protocol, which requires only T2W and DWI, is being widely tested for PCa diagnosis due to the rapid rise in the use of mp-MR for PCa. The biparametric MR (bp-MR) protocol is systematically used for PCa treatment, including T2W and DWI. The protocol of bp-MR can be executed of costly contrast medium for 15

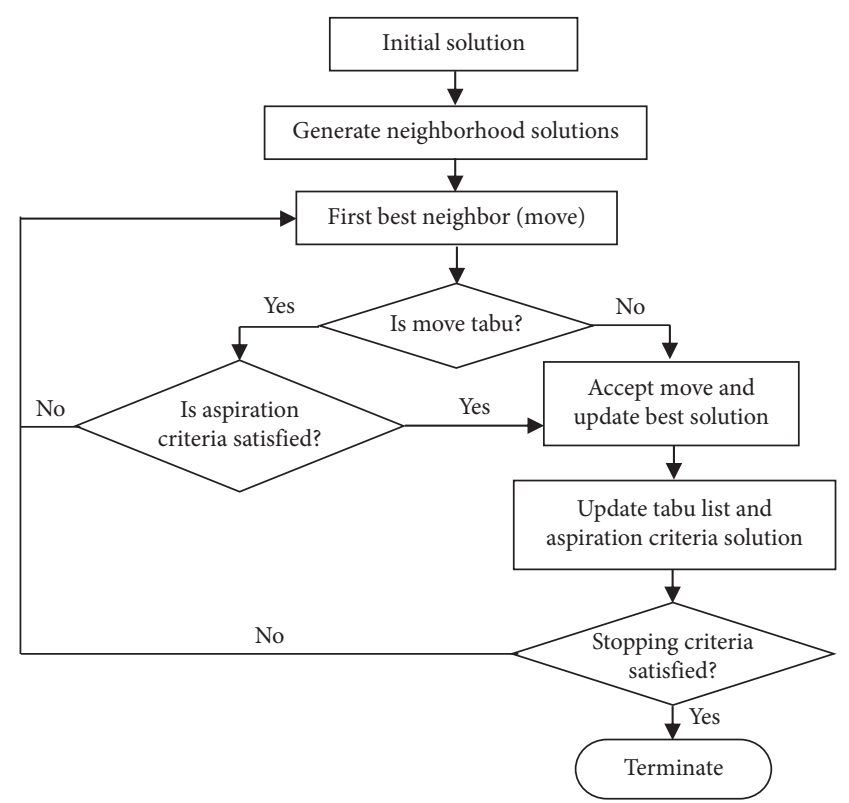

Figure 3: Flow chart of a short-term Tabu search [82].

minutes while retaining sufficient diagnostic reliability, all of which may facilitate the use and increase the efficiency $[60,61]$. Currently, many types of research focusing on bp$\mathrm{MR}$ or comparisons between bp-MR and mp-MR were published [61, 62]. In an article published in 2017, Greer et al. [63] suggested that the application of DCEI-MR could benefit the diagnostic of prostate cancer, because abnormal DCEI-MR findings improve the cancer detection rate in each of the PI-RADSv2 categories 2, 3, 4, and 5. However, those who advocated using bp-MR or opposed the use of DCEIMR suggested that compared with mp-MR.

Magnetic Resonance Fingerprinting (MRF). Magnetic Resonance Fingerprinting (MRF) is the newly invented mapmethod for nuclear magnetic resonance imaging introduced by $\mathrm{Ma}$ et al. [64] that could concurrently multiple approximate parameters of quantitative tissue properties, such as $\mathrm{T} 1$ and $\mathrm{T} 2$ relaxation times by transitory signal evolutions and data analysis. With this technology, MRF can provide a solution to this problem in effective and fast scan times by collecting quantitative measurements. Previous studies have shown that MR fingerprinting parameter maps have strong reproductivity in firm tissues in the supratentorial human brain region [65]. The support of larger multicenter, randomized control trials is still necessary if reproducibility and repeatability can be enhanced in PCa detection. Furthermore, 3D-1H-MRSI was also shown to be reproducible by Lage-maat et al. [66]. However, it was suggested that the reliability had been constrained by a small sample; further population-based experiments were also needed for proof of this in the future.

Challenges with Using MR Images. Despite the enhancement of the accuracy for PCa detection with mp-MR in the diagnostic process, the widespread use of $\mathrm{mp}-\mathrm{MR}$ for $\mathrm{PCa}$ diagnosis has been embedded by various challenges. 
Standardization. The features of the used scanner determine the output quality of Prostate Cancer detection using mpMRI (vendor, strength of the magnet field, technique, technology, etc.), patient factors (his movement, preparation etc.), and most significantly, the radiologists' interpretation [67].

Personal Challenges, Inter- and Intra-Reader Variability. Even with standardization of diagnosis and interpretation, there are certain drawbacks of perception, such as harmless circumstances, ERC-related artifacts, or changes in appearance the following care. High-level prostate mp-MR expertise is crucial to correct management that is not accessible in many centers $[2,68]$. The variance of inter-reader is also a problem for expert readers $[69,70]$.

The Cost. Multi-Parametric MR (mp-MR) is a rather costly analysis, or the costs are high. The mp-MR's ability to prevent biopsies, reduce overtreatment, and contributing to a higher quality of life can result in overall cost-effectiveness, but further studies are needed to confirm this [25, 71]. Reducing the mp-MR protocol to bp-MR could reduce the scanning time from 40 minutes to 15 minutes, avoid using the contrast medium, and reduce costs [60].

\section{Proposed Methodologies of CAD System}

We propose two research methodologies of CAD systems based on NIR, MIR, and MRI images in this work. We describe both methodologies in the following subsections.

\subsection{Research Methodology of CAD System on NIR and MIR} Images. This section describes the research methodology used on NIR and MIR images for designing an intelligent CAD system in detail. Figure 2 illustrates the feature extraction, infrared-bands feature selection, and classification phases for prostate cancer diagnosis. Mainly, we adopt a traditional pattern recognition approach, which involves the following stages:

(1) Inputs: The methodology supposes that the system receives the VIR, NIR, and MIR multispectral images from stroma samples, BPH, PIN, and PCa as inputs. These images are also used to measure and evaluate NIR and MIR technology's performance as benchmarks.

(2) Feature extraction: This stage is extremely crucial in extracting and obtaining relevant features for each band.

(3) Band selection: A selection method is conducted for band selection and removing redundant and irrelevant bands.

(4) Learning: Selected experts with supervised learning methods are used to learn NIR and MIR combined information after solving the curse-of-dimensionality issue through the feature selection method.

(5) Testing: in this stage, the new samples are tested and identified in the developed CAD system.
The system development will be deployed to create three main phases: (i) feature extraction phase, (ii) feature selection from useful bands, and (iii) classification phase. After obtaining multispectral images, important features are extracted from multispectral bands images. Then, the extracted features from useful bands are selected. Finally, the selected features are classified.

3.1.1. Feature Extraction. After multispectral image acquisition, essential features are extracted from NIR and MIR images. For this, three effective algorithms are used, which are the traditional first- and second-order Haralick texture features [72], the histogram-based features such as local binary pattern (LBP), and local phase quantization (LPQ). The following subsections describe these algorithms in detail.

NIR and MIR Local Binary Patterns (LBPs). The LBP was first introduced by Ojala et al. [73] as a gray-scale invariant pattern to measure texture feature in the source images. Its tolerance for any illumination changes characterizes it. Moreover, it is effective compared to other approaches, so that the images can be analyzed in real-time. Generally, the LBP operator is derived from the related information of texture on the local neighborhood. LBP can be extended to multispectral images and easy to handle multiscale analysis, which is quite efficient for prostate cancer classification applications.

NIR and MIR Local Phase Quantization. Local phase quantization (LPQ) [74] is another attractive and powerful texture descriptor for many real-world applications, such as image retrieval and face recognition. Its higher accuracy and computational complexity characterize it. LPQ uses the local phase information, which is computed in a window for every image position. LPQ is similar to LBP in its support for multiscale analysis and the ability to extend to multispectral image analysis.

3.1.2. Infrared-Multispectral Band Selection. In this work, we proposed the use of a considerable amount of NIR and MIR bands. For this regard, band selection is crucial as it is challenging to figure out which bands have the relevant information, or which two bands are redundant. Selecting the essential bands helps reduce the cost of feature extraction in test images. We dealt with band selection as the feature selection problem from the mathematical point of view. However, instead of handling the features, we search for the optimal number of bands, where some of the current algorithms for feature selection can be exploited.

Let $n$ be the number of multispectral bands extracted acquired from the NIR and MIR spectrum. We used sequential forward selection (SFS) [75] as landmine detection using multispectral images [76]. SFS is known to be a simple greedy sequential search algorithm. Another algorithm is the sequential forward floating search (SFFS) and sequential backward floating search (SBFS). Compared with SFS, the SFFS and SBFS algorithms are more efficient, especially 
finding optimal solutions for minor and medium-scale problems [77]. Nevertheless, SFFS and SBFS algorithms cannot converge to locally optimal solutions for large-scale issues when $n$ is more significant than $100[78,79]$. Recently, TS and genetic algorithms are used profusely to handle the problems of having an exponential and noisy search space with numerous local optima [79-82]. In this work, we aim to introduce the Tabu search for band selection. Tabu search is described in the following subsection.

Tabu Search: Tabu search was introduced by Glover [83]. Tabu Search (TS) can be described as a meta-heuristic, which is based on three principles: first, the use of data structure to memorize the history of the evolution of the search process; second, the use of a control mechanism to strike a balance between the acceptance, or not, of a new configuration, based on the information recorded in the taboo queue regarding the desired restrictions and aspirations; third, the intensification and diversification strategy procedures using short and long term memories. Accordingly, the TS algorithm is recommended to be used in the research methodology. The TS algorithm is given in Figure 3.

3.1.3. Classification. Previous works [82, 84-87] have been proposed for performing the classification task. In this section, we focus on investigating the methods for improving classification accuracy. The literature has already resulted in effective classifiers using a round-robin method, in which multiclass complex problems have been broken into many binary-class simple problems. Investigating the new classification techniques, such as logistic regression, Dempster-Shafer theory, decision template, and fuzzy integral method, is highlighted to determine each band's contribution of the NIR/MIR to the overall classification process. These new classification techniques are introduced below.

Logistic Regression. The logistic regression method transforms the classification problem into a regression problem $[88,89]$. The process tries different performances of distinct classifiers. It seems like a Borda count method in terms of generalization and assigning optimal linear weights to the rank scores for reflecting the significance of the separate classifiers in the combined decision.

Fuzzy Integral. For the diversity and capacity of different classifiers aggregation, the fuzzy integral has been applied to classifiers combinations $[90,91]$. A fuzzy measure is utilized to reflect the importance of classifiers from a complete set of learners regarding the class. Choquet fuzzy integral and Sugeno fuzzy integral are two essential types of fuzzy integrals. A fuzzy measure of a classifier is computed from several fuzzy densities to represent the particular importance of the classifier. The fuzzy measure can be consistent with these fuzzy densities. The classification result of a class $c$ is a cooperation between the evidence represented by the class $c$ in the different classifiers and competence defined by the fuzzy measure. The fuzzy measure vector might be specific for the current data sample and new for each class. If the ordering of the classifier support is the same, the two vectors of fuzzy measure will be only the same.

Decision Templates. In this type of supervised learning method, the classification results of distinctive classifiers for a given data test set can be arranged in a matrix called a decision profile (DP). The elements of the DP matrix $(c, l)$ are a classifier $c$ for class label $l$. The decision templates (DTs) combine the classifiers $[92,93]$, in which each decision template is computed from a test example given a trained classifier. A decision template of a class is the centroid of this class in the intermediate of the feature space. The anticipated decision profile of this class is related to this decision template. The prediction of a class label in a combination of classifiers can be calculated using a similarity measure (e.g., Euclidean distance) between the existing DP (of the test example to be classified) and the DTs classes.

Dempster-Shafer Combination. The Dempster-Shafer calculus $[94,95]$ is a method used for controlling the degrees of belief. Compared with the Bayesian approach, it is more general and does not involve adding additive probability. In [93], the author introduced a method that uses the theory of the Dempster-Shafer's evidence to combine the outputs of multiple classifiers and give a soft production. The training process of this approach is equal to the training of the decision templates approach. However, rather than computing the similarity between the decision profile and the decision template, the new data points are classified by calculating the proximities between each classifier output and decision template. For every classifier and every class, two basic probability assignments (BPAs) are constructed based on the proximities. By using the BPAs, the degrees of belief are computed based on the orthogonal sum rule. From the degrees of belief, the final degree of predictions is calculated for each class.

\subsection{Research Methodology of CAD System on MRI Images.} The use of MRI technology to diagnose PCa requires radiologists to interpret vast amounts of images and involves knowledge of experience that is not widely available. Automatic methods may simplify the radiologists' job, increasing read time and variance of the reader [62]. Automated approaches have been established to help less experienced readers reach the same performance level as professionals. Figure 4 shows a typical workflow of a computerized prostate cancer diagnosis system using $\mathrm{mp}-$ MR images.

3.2.1. MR Acquisition. The mp-MR analysis typically consists of an anatomical sequence (T2-weighted (T2W)) images and multiple functional sequences, generally DWI and DCE sequences. The sequence choice is based on the medical need and time and costs limitations [61]. There is currently growing research into the use of mp-MR as a triage tool. It is unlikely that patients with negative mp-MR will have any clinically meaningful PCa and could avoid biopsy. When using mp-MR as a triage test, $27 \%$ of patients are diagnosed 


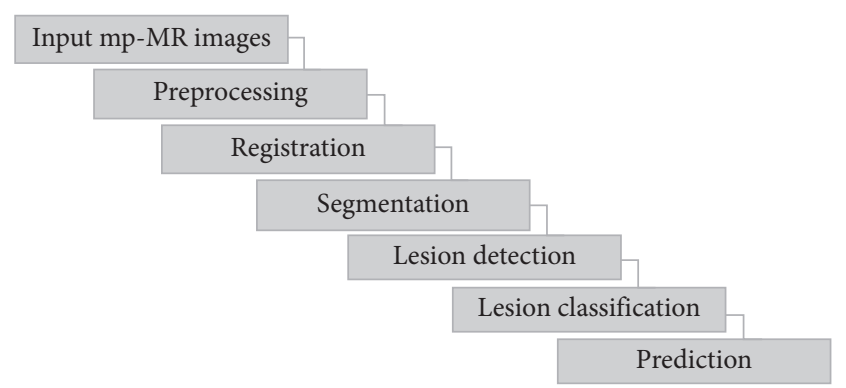

FIgURE 4: A typical workflow of automated prostate cancer diagnosis system using mp-MR images.

with a biopsy, and less than (5\%) are diagnosed with clinically insignificant PCa [94-96]. Therefore, young patients with elevated or reduced PSA must retain the normal TRUS $+\mathrm{B}$ until a definitive conclusion is reached on the negative predictive value of $\mathrm{mp}-\mathrm{MR}$ [97].

3.2.2. Preprocessing. Preprocessing the images requires standardization of image intensities, where the T2W image sequence, in particular, suffers from inter-and intra-patient variability, even for images collected using the same scanner and procedure. Other standard methods of preprocessing include noise filtering and correction of field bias [24]. The selection of steps to preprocess depends on the dataset and the software.

3.2.3. Registration. Registration is known as a method of aligning two or more images, may be helpful during the evaluation to compensate for patient movement and changes in bladder rectum filling. Long-term MR test procedures (e.g., DCE imaging) increase the likelihood of substantial patient movement and identify images $[3,15]$.

3.2.4. Segmentation. The segmentation process is a critical stage of the diagnosis process. The accurate diagnosis depends on the precise prostate segmentation from the obtained image [98-100]. The lack of clear boundaries and considerable variability in the prostate shape and appearance makes manual delineation a challenge. It is well known that the T2W image sequence offers the best analysis of prostate anatomy and the ability to delineate margins and distinguish between the zones of the prostate gland [101]. The manual delineation is very time-consuming and requires prostate MRI experience. In the literature, automated methods include map-based methods, model-based methods (e.g., effective form model), and edge-based methods [102]. In recent years, techniques have focused on the use of deep learning approaches, especially deep convolution neural networks (CNNs), that have made significant progress in the analysis of clinical images, including prostate segmentation [103]. Recently, more emphasis has been found on the segmentation techniques for prostate zones. Many $\mathrm{PCa}$ is located at the Peripheral Zone PZ, and since the PCa's biological activity varies between zones, this information is essential for making medical decisions $[62,104-106]$. Recent zonal segmentation studies have used various voxel identification approaches (3D pixel analog) and active form models [107-115]. The Current first place in the MICCAI Grand Prostate MRI segmentation challenge (PROSTATE12) is a CNN solution (reaching a coefficient of 0.8721 Dice score) [109]. One of the main challenges in zonal segmentation is the absence of gland apex and base characteristics and the gradients $[106,116]$.

3.2.5. Detection. The beginning was in 2003 with Chan et al. [117], the initial work on automated methods in prostate mp-MR focused on identifying suspect areas for directed guided biopsies of MR. The most common approach in the literature is to identify voxels as either PCa or normal tissue based on various image characteristics such as texture, signal strength, and data on gradients. A study conducted by Rampun et al. examined 215 T2WMR texture features for classifying $\mathrm{PZ}$ voxels as malignant and benign using 11 different classifiers (e.g., support vector machine (SVM), random woods, naïve Bayes, and $\mathrm{k}$-nearest neighbor) [118]. Combining the $\mathrm{T} 2 \mathrm{~W}$ sequence with one or more usable arrangements provides better detection over a single image mode. Extracted object characteristics from T2W, DCE and DWI resulted in AUC of 0.95 in a study by Peng et al. with using a linear discriminatory analysis to classify interest regions as either cancer or normal [67]. Nevertheless, most studies use T2WMR in conjunction with DWI, including ADC and/or DCE scanning. MRS was also investigated. Probably because of the difficulty and duration of data acquisition, the MRS has not achieved broad acceptance [119]. Various studies agree that a zone-aware Classifier significantly improves PCa [78, 120] identification. Many reported PCa detection algorithms to record an area between 0.80 and 0.89 under the receiver characteristic curve (AUC) [15]. One of the research showing the highest performance in literature is the analysis described by Peng et al. [67].

3.2.6. Classification. The selection of treatment for patients with PCa is based on clinical factors such as PSA, GS, age, and comorbidity. As previously mentioned, the GS is the most important indicator of disease progression, mortality, and outcomes. Since the GS of prostate biopsies sometimes varies from the true GS of RP, there is a medical need to distinguish slow-growing, indolent PCa better from those of RP with catastrophic outcomes of clinical significance [119]. The mp-MRI is usually used to determine PCa aggressiveness for noninvasive, pretreatment purposes. GS and ADC values are substantially associated with lower ADC values suggesting higher GS. Moreover, other studies found an association between DCE Parameters, frequency of T2W signal, and aggressiveness of PCa. Nevertheless, these single parameters are not enough to predict the GS alone [121-126]. Many studies have investigated algorithms with multiple image characteristics, such as shape, T2W, DWI and ADC frequency, to differentiate malignant from benign lesions or to classify lesions into clinically insignificant (GS6) or clinically insignificant. (GSB7) promising results [127-133]. Holtz et al. studied a small, medium, and high- 
grade three-class classifier compared to a two-class system and showed low performance for the three-class system. One analysis achieved accuracies of up to 0.93 for two-class classification of GS range versus GS range 7 , and $7(3+4)$ versus $7(4+3)$ using ADC and T2W derived feature [132]. The sensitivity of 100 percent and specificity of 76.92 percent has been achieved in a more recent study focused on a multimodal convolution neural network to distinguish GS6 from GS MUSK7 [129]. Because the prognosis and therapeutic options vary for each GS category, it would be of medical interest to determine lesions more accurately into more than two or three classes of clinical interest.

\section{Clinical Application}

4.1. Diagnosis. There is a high clinical need for developing fast and cost-effective computer diagnostic tools. Researchers have focused on this area, and there were plenty of attempts to build CAD automated systems for diagnosing prostate cancer using NIR, MIR, and MRS classification and supportive imaging types such as DCE-MR analysis. To increase diagnostic precision, the $\mathrm{MR}$ anatomic images (T2W) are combined with functional techniques because the single method cannot adequately detect and characterize PCa [119].

Chan et al. have one of the first attempts for implementing an mp-MR CAD system for the diagnosis of $\mathrm{PCa}$ using the approach of T2, T2W, and line scan to detect the predefined area of PZ of the PCa [117]. In the field of fuzzy logic techniques, Liu et al. [134] presented PCa detection using multispectral MR and NIR images. Moreover, Tiwari et al. [135] used the MRS with T2W-MRI to identify the presence of PCa by the existence of voxels affected by PCa.

But in the research work [67], Peng et al. combined 10th percentile ADC, average ADC, and T2W skewness to differentiate between normal and abnormal tissues of the prostate. In their approach, they obtained an AUC value of 0.95 .

Litjens et al. [136] developed a fully automated CAD system to recognize prostate cancer by performing two phases. The first is the detection phase that includes segmentation of prostate on T2W MRI, extracting features of the voxel, finally classification and select a candidate. After that, the second phase is responsible for performing processes on the output of phase one. In phase two, segmentation and feature extraction and classification for the candidate. Although the use of 347 patients data, the system reaches an AUC value of 0.889 .

4.2. Aggressiveness. It is important to construct an intelligent system for differentiating between high-grade cancer and indolent, slow-growing tumors based on initial PSA level, Gleason score, and patient age [137, 138]. One of the commonly employed statistical variables of $\mathrm{PCa}$ is the Gleason test, which utilizes a ranking system to determine the classification in PCa. Tumors of higher grades tend to grow rapidly and spread faster than tumors of lower grades.
Research by Yamamura et al. [139] found that the ADC values and the Gleason level were very strongly negatively correlated, although MRS displayed no significant correlation. PCa aggressively test approaches include noninvasive DWI, DCE-MRI, and MRS. Oto et al. [140] stated that the multi-parametric MR such as MR spectroscopy, T2weighted imaging, dynamic contrast-enhanced MR (DCEMRI), and diffusion-weighted imaging (DWI) is the best modality of imaging for staging and diagnosis of PCa. In vivo, MRS imaging showed an improvement in the Gleason level $[141,142]$ ratio (choline + creatine)/citrate. The magic angle spinning ex vivo (HRMAS) MRS [143] has also shown this relation. Nevertheless, there was no link between metabolite ratios and aggressiveness [144, 145] observed in other in vivo MRS imaging studies.

Changes in the signal intensity were associated with its aggressiveness [146] on the T2W-MRI for PCa detection. Lee et al. [147] demonstrated the possibility of improving the prediction of minor $\mathrm{PCa}$ in candidates for active monitoring treatment through simple measurements for the diameter of suspicious tumor lesions on DWI.

4.3. Biopsy Guidance. The biopsy may not accurately depict the whole gland, but examines a small portion of the prostate. The Gleason value in a random systemic biopsy, driven by TRUS, is usually believed to lower the test, since the biopsy may not have shown a less distinct trend $[148,149]$. Natural routine biopsies of TRUS also require multiple biopsy procedures combined with pain and possible disease [150]. Lesions have to be reliably identified, described, and treated during biopsy for minimizing overtreatment and amount of biopsies. In order to improve the detection rate of prostate biopsies, more successful imagingled guided biopsy approaches are being studied. In recent years, many minimally invasive, oriented approaches to conserve organ were used as additional options in contrast to the revolutionary use of PCa [150]. The following table (Table 1.) illustrates the studies in this field.

\section{Discussion, Observation, and Future Directions}

CAD diagnosis approaches to prostate cancer from medical imagery are based on various widely used NIR, MIR, MRI and TRUS dissecting methods. These are highly invasive and can assist in picture-orientated operations. They aim to detect prostate cancer early and can be used to enhance the remediation of patients. Nonetheless, problems remain that must be overcome to be used in the clinical environment so that a more efficient diagnostic method can be used. These include the following:

(1) Identifying the best imagery group that can precisely differentiate between benign and malignant groups, particularly with NIR, MIR, and multispectral MR such as 1-MR, T2-MR, DWI-MR, and DCE-MR, to design robust MR CAD systems (e.g., NIR, MIR, T1 MR, T2 MR, DWI MR, and DCE MR). Due to the increased diagnostic discrimination characteristics, 
TABLE 1: Summary of current studies in the literature.

\begin{tabular}{|c|c|c|c|c|c|c|}
\hline Researcher & Images type & Classifier & Region & Dataset size & Findings & $\begin{array}{c}\text { Published } \\
\text { date }\end{array}$ \\
\hline Chan et al. [117] & T2WI, ADC, T2 & SVM, FLD & $\mathrm{PZ}$ & 15 & $\begin{array}{c}\text { FLD, } A U C=0.839 ; S V M, \\
\text { AUC }=0.761\end{array}$ & 2003 \\
\hline Puech et al. [76] & DCE & $\begin{array}{l}\text { Software called } \\
\text { "ProCAD" }\end{array}$ & $\mathrm{PZ}, \mathrm{TZ}$ & 100 & $\begin{array}{c}\mathrm{Se} / \mathrm{Sp}=100 / 49 \% \mathrm{TZ}, \mathrm{Se} / \\
\mathrm{Sp}=100 / 40 \%\end{array}$ & 2007 \\
\hline $\begin{array}{l}\text { Tiwari et al. } \\
{[151]}\end{array}$ & MRS & Spectral clustering & WP & 14 & $\begin{array}{c}\mathrm{Se}=77.8 \%, \mathrm{FP}=28.92 \%, \\
\mathrm{FN}=0.88 \%\end{array}$ & 2007 \\
\hline Vos et al. [73] & DCE & SVM & $\mathrm{PZ}$ & 34 & $\mathrm{AUC}=0.83$ & 2008 \\
\hline $\begin{array}{l}\text { Viswanath et al. } \\
{[152]}\end{array}$ & DCE & Clustering & WP & 6 & $\mathrm{Se}=60.72 \%, \mathrm{sp}=83.24 \%$ & 2008 \\
\hline $\begin{array}{l}\text { Viswanath et al. } \\
\text { [153] }\end{array}$ & T2WI, DCE & Random forest & WP & 6 & $\mathrm{AUC}=0.815$ & 2009 \\
\hline Vos $[74]$ & DCE & SVM & $\mathrm{PZ}$ & 38 & $\mathrm{AUC}=0.80$ & 2009 \\
\hline Liu et al. [134] & $\mathrm{T} 2 \mathrm{~W}, \mathrm{~T} 2, \mathrm{ADC}, \mathrm{DCE}$ & Fuzzy MRF model & $\mathrm{PZ}$ & 11 & $\mathrm{Se}=89.58 \%, \mathrm{sp}=87.50 \%$ & 2009 \\
\hline $\begin{array}{l}\text { Tiwari et al. } \\
{[135]}\end{array}$ & MRS & NLDR & WP & 18 & $\mathrm{Se}=89.33 \%, \mathrm{sp}=79.79 \%$ & 2009 \\
\hline Vos et al. [154] & T2WI, DCE & SVM & $\mathrm{PZ}$ & 29 & $\mathrm{AUC}=0.89$ & 2010 \\
\hline Shah et al. [81] & T2WI, ADC, DCE & SVM & $\mathrm{PZ}$ & 31 & F-measure $=0.89$ & 2012 \\
\hline Liu et al. [155] & T2WI, ADC, and DCE & SVM & WP & 54 & $\mathrm{AUC}=0.82$ & 2013 \\
\hline Niaf et al. [59] & T2WI, ADC, and DCE $1.5 \mathrm{~T}$ & SVM & $\mathrm{PZ}$ & 30 & $\mathrm{AUC}=0.89$ & 2012 \\
\hline $\begin{array}{l}\text { Moradi et al. } \\
{[156]}\end{array}$ & DT, DCE $3 \mathrm{~T}$ & SVM & WP & 29 & AUC $=0.96$ & 2012 \\
\hline Niaf et al. [57] & T2WI, ADC, and DCE & P-SVM & WP & 49 & $\mathrm{AUC}=0.889$ & 2014 \\
\hline Peng et al. [67] & T2WI, ADC, and DCE $3 \mathrm{~T}$ & LDA & WP & 48 & $\mathrm{AUC}=0.95$ & 2013 \\
\hline $\begin{array}{l}\text { Artan et al. } \\
{[157]}\end{array}$ & T2WI, ADC, and DCE $1.5 \mathrm{~T}$ & CRF & $\mathrm{PZ}$ & 21 & $\mathrm{AUC}=0.79$ & 2010 \\
\hline $\begin{array}{l}\text { Tiwari et al. } \\
{[158]}\end{array}$ & T2WI, MRS $1.5 \mathrm{~T}$ & $\begin{array}{l}\text { SeSMiK-GE + random } \\
\text { forest }\end{array}$ & WP & 29 & $\mathrm{AUC}=0.89$ & 2013 \\
\hline $\begin{array}{l}\text { Tiwari et al. } \\
\text { [159] }\end{array}$ & T2WI, MRS $1.5 \mathrm{~T}$ & Random forest & WP & 36 & $\mathrm{AUC}=0.89$ & 2012 \\
\hline $\begin{array}{l}\text { Litjens et al. } \\
\text { [136] }\end{array}$ & $\begin{array}{c}\text { T2WI, PDWI, DCE, and } \\
\text { DWI } 3 T\end{array}$ & Random forest & WP & 347 & $\mathrm{AUC}=0.889$ & 2014 \\
\hline Molina et al. & T2WI, ADC, DCE & $\begin{array}{c}\text { Incremental learning } \\
\text { ensemble SVM }\end{array}$ & $\mathrm{PZ}$ & 12 & $\mathrm{Se}=84.4 \%, \mathrm{sp}=78.0 \%$ & 2014 \\
\hline $\begin{array}{l}\text { Kwak et al. } \\
{[161]}\end{array}$ & T2WI, DWI & SVM & $\begin{array}{l}\mathrm{PZ} \text { and } \\
\mathrm{TZ}\end{array}$ & 244 & AUC of 0.89 & 2015 \\
\hline Zhao et al. [162] & T2WI & ANN & $\begin{array}{l}\mathrm{PZ} \text { and } \\
\mathrm{CZ}\end{array}$ & 71 & $\begin{aligned} \mathrm{CG}, \mathrm{AUC} & =0.821 ; \mathrm{PZ}, \\
\mathrm{AUC} & =0.849\end{aligned}$ & 2015 \\
\hline Song et al. [26] & Multiparametric MRI & DCNN & $\mathrm{PZ}$ & $444+48+55$ & AUC of $94 \%$ & 2018 \\
\hline $\begin{array}{l}\text { Wang et al. } \\
{[163]}\end{array}$ & $\begin{array}{c}\text { Systematic } 12 \text {-core TRUS- } \\
\text { guided biopsies }\end{array}$ & CNNs based google net & WP & 360 & AUC of $96 \%$ & 2018 \\
\hline Sahrin [164] & Pelvis CT images & $\begin{array}{l}\text { Random forest, logistic } \\
\text { regression }\end{array}$ & WP & 182 & $96 \%$ accuracy & 2019 \\
\hline $\mathrm{Xu}$ et al. [165] & $\mathrm{T} 2 \mathrm{~W}, \mathrm{ADC}$ map & Residual networks & $\mathrm{PZ}, \mathrm{CZ}$ & 346 & AUC of $97 \%$ & 2019 \\
\hline
\end{tabular}

these systems are encouraging. However, because of the limited resolution of the different image modalities and the difference between slices between the acquired pictures, they are difficult. Therefore, they are relying on (i) develop difficult approaches to registration to coordinate imaging modalities effectively before disassembly and (ii) develop a robust segmentation algorithm applicable to the wide range of imaging methods.

(2) Develop algorithms for accurate prostate segmentation and tumor detection. These algorithms are still questionable due to (i) the nature of NIR, MIR, TRUS and MRI data, (ii) the similar intensity and proximity of adjacent nonprostatic tissue (e.g., the bladder), (iii) in many cases, DWI plays a major role in medical imaging. Applications such as this are to build an effective CAD imaging system for the detection of prostate cancer. This paper offered a summary of these systems covering noninvasive CAD systems from DWI. In this paper, we discussed current approaches for each step of machine-based learning CAD systems, with a focus on their advantages.

(3) Construct a successful and efficient, and intelligent CAD system that will reduce prostate cancer deaths by an earlier disease diagnosis. Researchers should 
then establish and test approaches to prostate diagnosis to enhance their results.

\section{Conclusion}

Since the 90s, they are constructing CAD systems have been recognized as one of the hottest fields for researches. Medical Precise and effective prostate cancer detection systems play a significant role in managing the mortality rate of serious malignancy among males. This paper presented an in-depth review of current computer-aided methods of a prostate cancer diagnosis. Mainly, the NIR, MIR, and MRI techniques have been discussed, which are used in the characterization of prostate tissue. In theory, the features of tissues by the NIR, MIR, and MRI images are essential for giving promising results of PCa detection, despite modeling interactions between these images and tissues. It is a significant achievement, though, with the inclusion of automatic ultrasound tissue characteristically methods, which are needed to correct pathological conditions with the acoustic properties of the tissue. It should be noted that intelligent computer-aided diagnostic systems are intended primarily to offer a second opinion and are not intended to replace a doctor. The rule also applies to prostate cancer. The occurrence of false-negative results, in this case specifically, means that a different perspective can be advantageous. If the biopsy is possible with this helping opinion, the biologist will select the biopsy sites.

\section{Abbreviations}

FMT: $\quad$ Fluorescent molecular tomographic

NIR: $\quad$ Near-Infrared

MIR: $\quad$ Mid-Infrared

MRI: Magnetic resonance imaging

PSMA: Prostate specific membrane antigen

FMT: $\quad$ Fluorescent molecular tomographic

AUC: Area under a receiver operating characteristic curve

FP: $\quad$ False positive

FN: $\quad$ False negative

Sp: $\quad$ Specificity

USPSTF: United states preventive services task force

FLD: $\quad$ Fisher linear discriminant

CI: $\quad$ Confidence interval

csCPa: Clinically significant prostate cancer

FLD: $\quad$ Fisher linear discriminant

OR: $\quad$ Odds ratio

PSA: Prostate-specific antigen

CRF: Conditional random fields

ROI: $\quad$ Region of interest

ADC: Apparent diffusion coefficient

CAD: $\quad$ Computer-aided diagnosis

DCE: Dynamic contrast-enhanced

LDA: Linear discriminant analysis

LLE: $\quad$ Locally linear embedding

MRS: Magnetic resonance spectroscopy

NLDR: Nonlinear dimensionality reduction

PCA: Principal component analysis
P-SVM: Probabilistic SVM

QDA: Quadratic discriminant analysis

Se: $\quad$ Sensitivity

SVM: Support vector machine

T1-PC: Principal component of T1-weighted dynamic series

T2WI: T2-weighted imaging

WP: Whole prostate

PZ: $\quad$ Peripheral zone

TZ: Transition zone

CZ: Central zone

LDA: Linear discriminant analysis

CG: $\quad$ Central gland

TRUS: Transrectal ultrasounds

STR: Stroma

BPH: $\quad$ Benign prostatic hyperplasia

PIN: Prostatic intraepithelial neoplasia.

\section{Data Availability}

No data were used to support the study.

\section{Conflicts of Interest}

The authors declare no conflicts of interest.

\section{Acknowledgments}

This project was funded by the National Plan for Science, Technology and Innovation (MAARIFAH), King Abdulaziz City for Science and Technology, Kingdom of Saudi Arabia, Award number 10-Bio-1905.

\section{References}

[1] S. Rizzo, G. Attard, and D. L. Hudson, "Prostate epithelial stem cells," Cell Proliferation, vol. 38, no. 6, pp. 363-374, 2005.

[2] J. C. Vilanova, Atlas of Multiparametric Prostate MRI, Springer, Berlin, Germany, 2018.

[3] R. L. Siegel, K. D. Miller, and A. Jemal, "Cancer statistics, 2019," CA: A Cancer Journal for Clinicians, vol. 69, no. 1, pp. 7-34, 2019.

[4] A. A. Bawazir, "Cancer incidence in Yemen from 1997 to 2011: a report from the Aden cancer registry," BMC Cancer, vol. 18, no. 1, p. 540, 2018.

[5] P. D. Baade, D. R. Youlden, S. M. Cramb, J. Dunn, and R. A. Gardiner, "Epidemiology of prostate cancer in the AsiaPacific region," Prostate International, vol. 1, no. 2, pp. 47-58, 2013.

[6] F. Bray, J.-S. Ren, E. Masuyer, and J. Ferlay, "Global estimates of cancer prevalence for 27 sites in the adult population in 2008," International Journal of Cancer, vol. 132, no. 5, pp. 1133-1145, 2013.

[7] F. H. Schröder and P. Boyle, "Screening for prostate cancerNecessity or nonsense?" European Journal of Cancer, vol. 29, no. 5, pp. 656-661, 1993.

[8] R. L. Siegel, K. D. Miller, and A. Jemal, "Cancer statistics, 2020," CA: A Cancer Journal for Clinicians, vol. 70, no. 1, pp. 7-30, 2020. 
[9] M. Moradi, P. Mousavi, and P. Abolmaesumi, "Computeraided diagnosis of prostate cancer with emphasis on ultrasound-based approaches: a review," Ultrasound in Medicine \& Biology, vol. 33, no. 7, pp. 1010-1028, 2007.

[10] F. H. Schröder, J Hugosson, M. J Roobol et al., "Prostatecancer mortality at 11 years of follow-up," The New England Journal of Medicine, vol. 366, no. 11, pp. 981-990, 2012.

[11] J. Hugosson, S. Carlsson, G. Aus et al., "Mortality results from the Göteborg randomised population-based prostatecancer screening trial," The Lancet Oncology, vol. 11, no. 8, pp. 725-732, 2010.

[12] C. Neppl-Huber, M. Zappa, J. W. Coebergh et al., "Changes in incidence, survival and mortality of prostate cancer in Europe and the United States in the PSA era: additional diagnoses and avoided deaths," Annals of Oncology, vol. 23, no. 5, pp. 1325-1334, 2012.

[13] G. L. Andriole, E. D Crawford, R. L Grubb et al., "Mortality results from a randomized prostate-cancer screening trial," The New England Journal of Medicine, vol. 360, no. 13, pp. 1310-1319, 2009.

[14] G. L. Andriole, E. D Crawford, R. L Grubb et al., "Prostate cancer screening in the randomized prostate, lung, colorectal, and ovarian cancer screening trial: mortality results after 13 years of follow-up," Journal of the National Cancer Institute, vol. 104, no. 2, pp. 125-132, 2012.

[15] S. Wang, "Computer aided-diagnosis of prostate cancer on multiparametric MRI: a technical review of current research," 2014.

[16] E. C. Serefoglu, "How reliable is 12-core prostate biopsy procedure in the detection of prostate cancer?" Canadian Urological Association Journal, vol. 7, no. 5-6, p. E293, 2013.

[17] J. C. Presti, J. J. Chang, V. Bhargava, and K. Shinohara, "The optimal systematic prostate biopsy scheme should include 8 rather than 6 biopsies: results of a prospective clinical trial," Journal of Urology, vol. 163, no. 1, pp. 163-167, 2000.

[18] N. E. Fleshner, M. O’Sullivan, and W. R. Fair, "Prevalence and predictors of a positive repeat transrectal ultrasound guided needle biopsy of the prostate," Journal of Urology, vol. 158, no. 2, pp. 505-509, 1997.

[19] M. Norberg, L. Egevad, L. Holmberg, P. Sparén, B. J. Norlén, and C. Busch, "The sextant protocol for ultrasound-guided core biopsies of the prostate underestimates the presence of cancer," Urology, vol. 50, no. 4, pp. 562-566, 1997.

[20] R. Llobet, J. Perezcortes, A. Toselli, and A. Juan, "Computeraided detection of prostate cancer," International Journal of Medical Informatics, vol. 76, no. 7, pp. 547-556, 2007.

[21] J. Nakashima, A. Tanimoto, Y. Imai et al., "Endorectal MRI for prediction of tumor site, tumor size, and local extension of prostate cancer," Urology, vol. 64, no. 1, pp. 101-105, 2004.

[22] D. Amsellem-Ouazana, P. Younes, S. Conquy et al., "Negative prostatic biopsies in patients with a high risk of prostate cancer," European Urology, vol. 47, no. 5, pp. 582-586, 2005.

[23] A. E. Wefer, H. Hricak, D. B. Vigneron et al., "Sextant localization of prostate cancer: comparison of sextant biopsy, magnetic resonance imaging and magnetic resonance spectroscopic imaging with step section histology," Journal of Urology, vol. 164, no. 2, pp. 400-404, 2000.

[24] N. R. Paterson, L. T Lavallée, L. N Nguyen et al., "Prostate volume estimations using magnetic resonance imaging and transrectal ultrasound compared to radical prostatectomy specimens," Canadian Urological Association journal = Journal de l'Association des urologues du Canada, vol. 10, no. 7-8, pp. 264-268, 2016.
[25] M. R. Pokorny, M. de Rooij, E. Duncan et al., "Prospective study of diagnostic accuracy comparing prostate cancer detection by transrectal ultrasound-guided biopsy versus magnetic resonance (MR) imaging with subsequent MRguided biopsy in men without previous prostate biopsies," European Urology, vol. 66, no. 1, pp. 22-29, 2014.

[26] Y. Song, Y.-D. Zhang, X. Yan et al., "Computer-aided diagnosis of prostate cancer using a deep convolutional neural network from multiparametric MRI," Journal of Magnetic Resonance Imaging, vol. 48, no. 6, pp. 1570-1577, 2018.

[27] M. Treviño, B Turkbey, B. J. Wood et al., "Rapid perceptual processing in two- and three-dimensional prostate images," Journal of Medical Imaging, vol. 2, Article ID 022406, 2020.

[28] P. Chirra, P. Leo, M. Yim et al., "Multisite evaluation of radiomic feature reproducibility and discriminability for identifying peripheral zone prostate tumors on MRI," J Journal of Medical Imaging, vol. 2, Article ID 024502, 2019.

[29] L. Zhen, X Liu, C Yegang et al., "Accuracy of multiparametric magnetic resonance imaging for diagnosing prostate cancer: a systematic review and meta-analysis," BMC Cancer, vol. 19, no. 1, pp. 1244-1315, 2019.

[30] J. O. Barentsz, J. Richenberg, R. Clements et al., "ESUR prostate MR guidelines 2012," European Radiology, vol. 22, no. 4, pp. 746-757, 2012.

[31] J. C. Weinreb, "PI-RADS prostate imaging-reporting and data system," 2015.

[32] P. C. Vos, J. O. Barentsz, N. Karssemeijer, and H. J. Huisman, "Automatic computer-aided detection of prostate cancer based on multiparametric magnetic resonance image analysis," Physics in Medicine and Biology, vol. 57, no. 6, pp. 1527-1542, 2012.

[33] E. Niaf, C. Lartizien, F. Bratan et al., "Prostate focal peripheral zone lesions: characterization at multiparametric MR imaging-influence of a computer-aided diagnosis system," Radiology, vol. 271, no. 3, pp. 761-769, 2014.

[34] V. Shah, B. Turkbey, H. Mani et al., "Decision support system for localizing prostate cancer based on multiparametric magnetic resonance imaging," Medical Physics, vol. 39, no. 7Part1, pp. 4093-4103, 2012.

[35] E. Niaf, O. Rouvière, F. Mège-Lechevallier, F. Bratan, and C. Lartizien, "Computer-aided diagnosis of prostate cancer in the peripheral zone using multiparametric MRI," Physics in Medicine and Biology, vol. 57, no. 12, pp. 3833-3851, 2012.

[36] O. Akin, E. Sala, C. S. Moskowitz et al., "Transition zone prostate cancers: features, detection, localization, and staging at endorectal MR imaging," Radiology, vol. 239, no. 3, pp. 784-792, 2006.

[37] H. Li, K. Sugimura, Y. Kaji et al., "Conventional MRI capabilities in the diagnosis of prostate cancer in the transition zone," American Journal of Roentgenology, vol. 186, no. 3, pp. 729-742, 2006.

[38] S. E. Viswanath, N. B. Bloch, J. C. Chappelow et al., "Central gland and peripheral zone prostate tumors have significantly different quantitative imaging signatures on 3 tesla endorectal, in vivo T2-weighted MR imagery," Journal of Magnetic Resonance Imaging, vol. 36, no. 1, pp. 213-224, 2012.

[39] C. M. A. Hoeks, T. Hambrock, D. Yakar et al., "Transition zone prostate cancer: detection and localization with 3-T multiparametric MR imaging," Radiology, vol. 266, no. 1, pp. 207-217, 2013.

[40] W. Liu, B. Turkbey, J. Sénégas et al., “AcceleratedT2mapping for characterization of prostate cancer," Magnetic Resonance in Medicine, vol. 65, no. 5, pp. 1400-1406, 2011. 
[41] F. I. Yamauchi, T. Penzkofer, A. Fedorov et al., "Prostate cancer discrimination in the peripheral zone with a reduced field-of-view T2-mapping MRI sequence," Magnetic Resonance Imaging, vol. 33, no. 5, pp. 525-530, 2015.

[42] X. Liu and I. S. Yetik, "Automated prostate cancer localization without the need for peripheral zone extraction using multiparametric MRI," Medical Physics, vol. 38, no. 6Part1, pp. 2986-2994, 2011.

[43] Y. Peng, Y. Jiang, C. Yang et al., "Quantitative analysis of multiparametric prostate MR images: differentiation between prostate cancer and normal tissue and correlation with gleason score-A computer-aided diagnosis development study," Radiology, vol. 267, no. 3, pp. 787-796, 2013.

[44] S. B. Sung-Bae Cho and J. H. Kim, "Combining multiple neural networks by fuzzy integral for robust classification," IEEE Transactions on Systems, Man, and Cybernetics, vol. 25, no. 2, pp. 380-384, 1995.

[45] J. Nowak, U. Malzahn, A. D. Baur et al., "The value of ADC, $\mathrm{T} 2$ signal intensity, and a combination of both parameters to assess Gleason score and primary Gleason grades in patients with known prostate cancer," Acta Radiologica, vol. 57, no. 1, pp. 107-114, 2016.

[46] P. S. Tofts, G. Brix, D. L. Buckley et al., "Estimating kinetic parameters from dynamic contrast-enhanced t1-weighted MRI of a diffusable tracer: standardized quantities and symbols," Journal of Magnetic Resonance Imaging, vol. 10, no. 3, pp. 223-232, 1999.

[47] A. Fedorov, J. Fluckiger, G. D. Ayers et al., “A comparison of two methods for estimating DCE-MRI parameters via individual and cohort based AIFs in prostate cancer: a step towards practical implementation," Magnetic Resonance Imaging, vol. 32, no. 4, pp. 321-329, 2014.

[48] A. S. Gliozzi, S. Mazzetti, P. P. Delsanto, D. Regge, and M. Stasi, "Phenomenological universalities: a novel tool for the analysis of dynamic contrast enhancement in magnetic resonance imaging," Physics in Medicine and Biology, vol. 56, no. 3, pp. 573-586, 2011.

[49] S. Mazzetti, A. S. Gliozzi, C. Bracco, F. Russo, D. Regge, and M. Stasi, "Comparison between PUN and Tofts models in the quantification of dynamic contrast-enhanced MR imaging," Physics in Medicine and Biology, vol. 57, no. 24, pp. 84438453, 2012.

[50] P. C. Vos, T. Hambrock, C. A. Hulsbergen - van de Kaa, J. J. Fütterer, J. O. Barentsz, and H. J. Huisman, "Computerized analysis of prostate lesions in the peripheral zone using dynamic contrast enhanced MRI," Medical Physics, vol. 35, no. 3, pp. 888-899, 2008.

[51] P. C. Vos, "Automated calibration for computerized analysis of prostate lesions using pharmacokinetic magnetic resonance images," in Proceedings of the International Conference on Medical Image Computing and Computer-Assisted Intervention, Springer, London, UK, September 2009.

[52] P. Puech, N. Betrouni, N. Makni, A.-S. Dewalle, A. Villers, and L. Lemaitre, "Computer-assisted diagnosis of prostate cancer using DCE-MRI data: design, implementation and preliminary results," International Journal of Computer Assisted Radiology and Surgery, vol. 4, no. 1, pp. 1-10, 2009.

[53] P. Puech, N. Betrouni, R. Viard, A. Villers, X. Leroy, and L. Lemaitre, "Prostate cancer computer-assisted diagnosis software using dynamic contrast-enhanced MRI," in Proceedings of the 2007 29th Annual International Conference of the IEEE Engineering in Medicine and Biology Society, IEEE, Lyon, France, August 2007.
[54] A. R. Padhani, C. J. Gapinski, D. A. Macvicar et al., "Dynamic contrast enhanced MRI of prostate cancer: correlation with morphology and tumour stage, histological grade and PSA," Clinical Radiology, vol. 55, no. 2, pp. 99-109, 2000.

[55] N. Dikaios, J. Alkalbani, M. Abd-Alazeez et al., "Zonespecific logistic regression models improve classification of prostate cancer on multi-parametric MRI," European Radiology, vol. 25, no. 9, pp. 2727-2737, 2015.

[56] J. Toivonen, H. Merisaari, M. Pesola et al., "Mathematical models for diffusion-weighted imaging of prostate cancer using b values up to $2000 \mathrm{~s} / \mathrm{mm} 2$ : correlation with Gleason score and repeatability of region of interest analysis," Magnetic Resonance in Medicine, vol. 74, no. 4, pp. 11161124, 2015.

[57] L. Boesen, E. Chabanova, V. Løgager, I. Balslev, and H. S. Thomsen, "Apparent diffusion coefficient ratio correlates significantly with prostate cancer gleason score at final pathology," Journal of Magnetic Resonance Imaging, vol. 42, no. 2, pp. 446-453, 2015.

[58] Y. Mazaheri, A. Shukla-Dave, H. Hricak et al., "Prostate cancer: identification with combined diffusion-weighted MR imaging and 3D1H MR spectroscopic imaging-correlation with pathologic Findings1," Radiology, vol. 246, no. 2, pp. 480-488, 2008.

[59] L. Boesen, N. Nørgaard, V. Løgager et al., "Assessment of the diagnostic accuracy of biparametric magnetic resonance imaging for prostate cancer in biopsy-naive men," JAMA Network Open, vol. 1, no. 2, p. e180219, 2018.

[60] I. Jambor, P. J. Boström, P. Taimen et al., "Novel biparametric MRI and targeted biopsy improves risk stratification in men with a clinical suspicion of prostate cancer (IMPROD trial)," Journal of Magnetic Resonance Imaging, vol. 46, no. 4, pp. 1089-1095, 2017.

[61] M. Fascelli, S. Rais-Bahrami, S. Sankineni et al., "Combined biparametric prostate magnetic resonance imaging and prostate-specific antigen in the detection of prostate cancer: a validation study in a biopsy-naive patient population," Urology, vol. 88, pp. 125-134, 2016.

[62] M. D. Greer, J. H. Shih, N. Lay et al., "Validation of the dominant sequence paradigm and role of dynamic contrastenhanced imaging in PI-RADS version 2," Radiology, vol. 285, no. 3, pp. 859-869, 2017.

[63] D. Ma, V. Gulani, N. Seiberlich et al., "Magnetic resonance fingerprinting," Nature, vol. 495, no. 7440, pp. 187-192, 2013.

[64] G. Körzdörfer, R. Kirsch, K. Liu et al., "Reproducibility and repeatability of MR fingerprinting relaxometry in the human brain," Radiology, vol. 292, no. 2, pp. 429-437, 2019.

[65] M. W. Lagemaat, C. M. Zechmann, J. J. Fütterer et al., "Reproducibility of 3D 1H MR spectroscopic imaging of the prostate at 1.5T," Journal of Magnetic Resonance Imaging, vol. 35, no. 1, pp. 166-173, 2012.

[66] A. R. Padhani, J. Weinreb, A. B. Rosenkrantz, G. Villeirs, B. Turkbey, and J. Barentsz, "Prostate imaging-reporting and data system steering committee: PI-RADS v2 status update and future directions," European Urology, vol. 75, no. 3, pp. 385-396, 2019.

[67] V. Panebianco, F. Barchetti, J. Barentsz et al., "Pitfalls in interpreting mp-MRI of the prostate: a pictorial review with pathologic correlation," Insights Into Imaging, vol. 6, no. 6, pp. 611-630, 2015.

[68] C. Jensen, Prostate Cancer Diagnosis Using Magnetic Resonance Imaging-A Machine Learning Approach, Aalborg Universitetsforlag, Aalborg, Denmark, 2018. 
[69] R. T. Gupta and A. B. Rosenkrantz, "Prostate MRI can be accurate but can variability be reduced?" Nature Reviews Urology, vol. 15, no. 6, pp. 339-340, 2018.

[70] J. Walz, "The "PROMIS" of magnetic resonance imaging cost effectiveness in prostate cancer diagnosis?" 2018.

[71] R. M. Haralick, K. Shanmugam, and I. H. Dinstein, "Textural features for image classification," IEEE Transactions on Systems, Man, and Cybernetics, vol. SMC-3, no. 6, pp. 610621, 1973.

[72] T. Ojala, M. Pietikainen, and T. Maenpaa, "Multiresolution gray-scale and rotation invariant texture classification with local binary patterns," IEEE Transactions on Pattern Analysis and Machine Intelligence, vol. 24, no. 7, pp. 971-987, 2002.

[73] V. Ojansivu and J. Heikkilä, "Blur insensitive texture classification using local phase quantization," in Proceedings of the International conference on image and signal processing, pp. 236-243, Cherbourg-Octeville, France, July 2008.

[74] A. W. Whitney, "A direct method of nonparametric measurement selection," IEEE Transactions on Computers, vol. C-20, no. 9, pp. 1100-1103, 1971.

[75] G. A. Clark, S. K. Sengupta, W. D. Aimonetti, F. Roeske, and J. G. Donetti, "Multispectral image feature selection for land mine detection," IEEE Transactions on Geoscience and Remote Sensing, vol. 38, no. 1, pp. 304-311, 2000.

[76] P. Pudil, J. Novovičová, and J. Kittler, "Floating search methods in feature selection," Pattern Recognition Letters, vol. 15, no. 11, pp. 1119-1125, 1994.

[77] M. Kudo and J. Sklansky, "Comparison of algorithms that select features for pattern classifiers," Pattern Recognition, vol. 33, no. 1, pp. 25-41, 2000.

[78] H. Zhang and G. Sun, "Feature selection using tabu search method," Pattern Recognition, vol. 35, no. 3, pp. 701-711, 2002.

[79] W. Siedlecki and J. Sklansky, "A note on genetic algorithms for large-scale feature selection," in Handbook of Pattern Recognition and Computer Vision, pp. 88-107, Springer, Berlin, Germany, 1993.

[80] S. O. Shim and T. S. Choi, "A novel iterative shape from focus algorithm based on combinatorial optimization," Pattern Recognition, vol. 43, no. 10, pp. 3338-3347, 2010.

[81] M. A. Tahir, A. Bouridane, and M. A. Roula, "Prostate cancer classification using multispectral imagery and metaheuristics," Computational Intelligence in Medical Imaging: Techniques and Applications, vol. 139, 2009.

[82] F. Glover and M. Laguna, "Tabu search," in Handbook of Combinatorial Optimization, pp. 2093-2229, Springer, Boston, MA, USA, 1998.

[83] M. A. Roula, "Machine vision and texture analysis for the automated identification of tissue patterns in prostatic tumours," Ph. D. thesis, Queens University of Belfast, Belfast, Northern Ireland, 2004.

[84] M. A. Tahir, A. Bouridane, F. Kurugollu, and A. Amira, "A novel prostate cancer classification technique using intermediate memory tabu search," EURASIP Journal on Advances in Signal Processing, vol. 2005, no. 14, pp. 1-9, 2005.

[85] M. A. Tahir and A. Bouridane, "Novel round-robin tabu search algorithm for prostate cancer classification and diagnosis using multispectral imagery," IEEE Transactions on Information Technology in Biomedicine, vol. 10, no. 4, pp. 782-793, 2006.

[86] S. Bouatmane, M. A. Roula, A. Bouridane, and S. AlMaadeed, "Round-Robin sequential forward selection algorithm for prostate cancer classification and diagnosis using multispectral imagery," Machine Vision and Applications, vol. 22, no. 5, pp. 865-878, 2011.

[87] T. K. A Ho, J. J. Hull, and S. N. Srihari, "Decision combination in multiple classifier systems," IEEE Transactions on Pattern Analysis and Machine Intelligence, vol. 16, no. 1, pp. 66-75, 1994.

[88] A. Saranll, "A unifying theory for rank-based multiple classifier systems, with applications in speaker identification and speech recognition," 2000.

[89] P. D. Gader, M. A. Mohamed, and J. M. Keller, "Fusion of handwritten word classifiers," Pattern Recognition Letters, vol. 17 , no. 6, pp. 577-584, 1996.

[90] L. I. Kuncheva, J. C. Bezdek, and R. P. W. Duin, "Decision templates for multiple classifier fusion: an experimental comparison," Pattern Recognition, vol. 34, no. 2, pp. 299-314, 2001.

[91] L. I. Kuncheva, "Using measures of similarity and inclusion for multiple classifier fusion by decision templates," Fuzzy Sets and Systems, vol. 122, no. 3, pp. 401-407, 2001.

[92] G. Shafer, A Mathematical Theory of Evidence, Princeton university press, Princeton, NJ, USA, 1976.

[93] A. P. Dempster, "A generalization of Bayesian inference," Journal of the Royal Statistical Society: Series B (Methodological), vol. 30, no. 2, pp. 205-232, 1968.

[94] A. Rhudd, J. McDonald, M. Emberton, and V. Kasivisvanathan, "The role of the multiparametric MRI in the diagnosis of prostate cancer in biopsy-naïve men," Current Opinion in Urology, vol. 27, no. 5, pp. 488-494, 2017.

[95] R. Renard-Penna, M. Roupret, E. Compérat et al., "Relationship between non-suspicious MRI and insignificant prostate cancer: results from a monocentric study," World Journal of Urology, vol. 34, no. 5, pp. 673-678, 2016.

[96] H. U. Ahmed, A. El-Shater Bosaily, L. C. Brown et al., "Diagnostic accuracy of multi-parametric MRI and TRUS biopsy in prostate cancer (PROMIS): a paired validating confirmatory study," The Lancet, vol. 389, no. 10071, pp. 815-822, 2017.

[97] V. Panebianco, G. Barchetti, G. Simone et al., "Negative multiparametric magnetic resonance imaging for prostate cancer: what's next?" European Urology, vol. 74, no. 1, pp. $48-54,2018$.

[98] G. Garg and M. Juneja, "Cancer detection with prostate zonal segmentation-a review," in Proceedings of the International Conference on Computing and Communication Systems, Springer, Nagoya, Japan, April 2018.

[99] G. Litjens, R. Toth, W. van de Ven et al., "Evaluation of prostate segmentation algorithms for MRI: the PROMISE12 challenge," Medical Image Analysis, vol. 18, no. 2, pp. 359-373, 2014.

[100] S. H. Park, Y. Gao, Y. Shi, and D. Shen, "Interactive prostate segmentation using atlas-guided semi-supervised learning and adaptive feature selection," Medical Physics, vol. 41, no. 11, p. 111715, 2014.

[101] M. L. Palmeri, Z. A. Miller, T. J. Glass et al., "B-mode and acoustic radiation Force impulse (ARFI) imaging of prostate zonal anatomy," Ultrasonic Imaging, vol. 37, no. 1, pp. 22-41, 2015.

[102] S. Ghose, A. Oliver, R. Martí et al., "A survey of prostate segmentation methodologies in ultrasound, magnetic resonance and computed tomography images," Computer Methods and Programs in Biomedicine, vol. 108, no. 1, pp. 262-287, 2012. 
[103] D. Shen, G. Wu, and H.-I. Suk, "Deep learning in medical image analysis," Annual Review of Biomedical Engineering, vol. 19, no. 1, pp. 221-248, 2017.

[104] J. J. Lee, I.-C. Thomas, R. Nolley, M. Ferrari, J. D. Brooks, and J. T. Leppert, "Biologic differences between peripheral and transition zone prostate cancer," The Prostate, vol. 75, no. 2, pp. 183-190, 2015.

[105] H. R. Ehrenberg, "Decision forests for learning prostate cancer probability maps from multiparametric MRI," in Proceedings of the Medical Imaging 2016: Computer-Aided Diagnosis, San Diego, CA, USA, March 2016.

[106] H. A. Vargas, O. Akin, T. Franiel et al., "Normal central zone of the prostate and central zone involvement by prostate cancer: clinical and MR imaging implications," Radiology, vol. 262, no. 3, pp. 894-902, 2012.

[107] N. Makni, A. Iancu, O. Colot, P. Puech, S. Mordon, and N. Betrouni, "Zonal segmentation of prostate using multispectral magnetic resonance images," Medical Physics, vol. 38, no. 11, pp. 6093-6105, 2011.

[108] G. Litjens, "A pattern recognition approach to zonal segmentation of the prostate on MRI," in Proceedings of the International Conference on Medical Image Computing and Computer-Assisted Intervention, Springer, Nice, France, October 2012.

[109] R. Toth, J. Ribault, J. Gentile, D. Sperling, and A. Madabhushi, "Simultaneous segmentation of prostatic zones using active appearance models with multiple coupled levelsets," Computer Vision and Image Understanding, vol. 117, no. 9, pp. 1051-1060, 2013.

[110] Y. Chi, "A compact method for prostate zonal segmentation on multiparametric MRIs," in Proceedings of the Medical Imaging 2014: Image-Guided Procedures, Robotic Interventions, and Modeling, San Diego, CA, USA, September 2014.

[111] O. Chilali, P. Puech, S. Lakroum, M. Diaf, S. Mordon, and N. Betrouni, "Gland and zonal segmentation of prostate on T2W MR images," Journal of Digital Imaging, vol. 29, no. 6, pp. 730-736, 2016.

[112] T. Clark, J Zhang, S Baig, A Wong, M. A Haider, and F Khalvati, "Fully automated segmentation of prostate whole gland and transition zone in diffusion-weighted MRI using convolutional neural networks," Journal of Medical Imaging (Bellingham, Wash.), vol. 4, no. 4, Article ID 041307, 2017.

[113] J. Zhang, "A local ROI-specific atlas-based segmentation of prostate gland and transitional zone in diffusion MRI," Journal of Computational Vision and Imaging Systems, vol. 2, no. 1, 2016.

[114] W. Qiu, J. Yuan, E. Ukwatta, Y. Sun, M. Rajchl, and A. Fenster, "Dual optimization based prostate zonal segmentation in 3D MR images," Medical Image Analysis, vol. 18, no. 4, pp. 660-673, 2014.

[115] W. Qiu, "Efficient 3D multi-region prostate MRI segmentation using dual optimization," in Proceedings of the International Conference on Information Processing in Medical Imaging, Springer, Asilomar, CA, USA, July 2013.

[116] Q. Zhu, "Exploiting interslice correlation for MRI prostate image segmentation, from recursive neural networks aspect," 2018.

[117] I. Chan, W. Wells, R. V. Mulkern et al., "Detection of prostate cancer by integration of line-scan diffusion, T2mapping and T2-weighted magnetic resonance imaging; a multichannel statistical classifier," Medical Physics, vol. 30, no. 9, pp. 2390-2398, 2003.

[118] A. Rampun, L. Zheng, P. Malcolm, B. Tiddeman, and R. Zwiggelaar, "Computer-aided detection of prostate cancer in T2-weighted MRI within the peripheral zone," Physics in Medicine and Biology, vol. 61, no. 13, pp. 4796-4825, 2016.

[119] L. Liu, Z. Tian, Z. Zhang, and B. Fei, "Computer-aided detection of prostate cancer with MRI," Academic Radiology, vol. 23, no. 8, pp. 1024-1046, 2016.

[120] S. B. Ginsburg, A. Algohary, S. Pahwa et al., "Radiomic features for prostate cancer detection on MRI differ between the transition and peripheral zones: preliminary findings from a multi-institutional study," Journal of Magnetic Resonance Imaging, vol. 46, no. 1, pp. 184-193, 2017.

[121] T. Hambrock, D. M. Somford, H. J. Huisman et al., "Relationship between apparent diffusion coefficients at 3.0-T MR imaging and Gleason grade in peripheral zone prostate cancer," Radiology, vol. 259, no. 2, pp. 453-461, 2011.

[122] Y.-J. Chen, W.-C. Chu, Y.-S. Pu, S.-C. Chueh, C.-T. Shun, and W.-Y. I. Tseng, "Washout gradient in dynamic contrastenhanced MRI is associated with tumor aggressiveness of prostate cancer," Journal of Magnetic Resonance Imaging, vol. 36, no. 4, pp. 912-919, 2012.

[123] O. Starobinets, J. P. Simko, K. Kuchinsky et al., "Characterization and stratification of prostate lesions based on comprehensive multiparametric MRI using detailed wholemount histopathology as a reference standard," NMR in Biomedicine, vol. 30, no. 12, Article ID e3796, 2017.

[124] C. A. Woodfield, G. A. Tung, D. J. Grand, J. A. Pezzullo, J. T. Machan, and J. F. Renzulli, "Diffusion-weighted MRI of peripheral zone prostate cancer: comparison of tumor apparent diffusion coefficient with Gleason score and percentage of tumor on core biopsy," American Journal of Roentgenology, vol. 194, no. 4, pp. W316-W322, 2010.

[125] S. Barbieri, M. Brönnimann, S. Boxler, P. Vermathen, and H. C. Thoeny, "Differentiation of prostate cancer lesions with high and with low Gleason score by diffusion-weighted MRI," European Radiology, vol. 27, no. 4, pp. 1547-1555, 2017.

[126] G. Nketiah, M. Elschot, E. Kim et al., “T2-weighted MRIderived textural features reflect prostate cancer aggressiveness: preliminary results," European Radiology, vol. 27, no. 7, pp. 3050-3059, 2017.

[127] P. Sobecki, "Feature extraction optimized for prostate lesion classification," in Proceedings of the 9th International Conference on Bioinformatics and Biomedical Technology, Lisbon, Portugal, May 2017.

[128] J. N. Holtz, R. K. Silverman, K. J. Tay et al., "New prostate cancer prognostic grade group (PGG): can multiparametric MRI (mpMRI) accurately separate patients with low-, intermediate-, and high-grade cancer?" Abdominal Radiology, vol. 43, no. 3, pp. 702-712, 2018.

[129] M. H. Le, J. Chen, L. Wang et al., "Automated diagnosis of prostate cancer in multi-parametric MRI based on multimodal convolutional neural networks," Physics in Medicine \& Biology, vol. 62, no. 16, pp. 6497-6514, 2017.

[130] A. Wibmer, H. Hricak, T. Gondo et al., "Haralick texture analysis of prostate MRI: utility for differentiating noncancerous prostate from prostate cancer and differentiating prostate cancers with different Gleason scores," European Radiology, vol. 25, no. 10, pp. 2840-2850, 2015.

[131] J. Li, Z. Weng, H. Xu et al., "Support Vector Machines (SVM) classification of prostate cancer Gleason score in central gland using multiparametric magnetic resonance images: a cross-validated study," European Journal of Radiology, vol. 98, pp. 61-67, 2018.

[132] D. Fehr, H. Veeraraghavan, A. Wibmer et al., "Automatic classification of prostate cancer Gleason scores from 
multiparametric magnetic resonance images," Proceedings of the National Academy of Sciences, vol. 112, no. 46, pp. E6265-E6273, 2015.

[133] P. Sobecki, D. Życka-Malesa, I. Mykhalevych, K. Sklinda, and A. Przelaskowski, "MRI imaging texture features in prostate lesions classification," in EMBEC \& NBC 2017, pp. 827-830, Springer, Berlin, Germany, 2017.

[134] X. Liu, D. L. Langer, M. A. Haider, Y. Yang, M. N. Wernick, and I. S. Yetik, "Prostate cancer segmentation with simultaneous estimation of Markov random field parameters and class," IEEE Transactions on Medical Imaging, vol. 28, no. 6, pp. 906-915, 2009.

[135] P. Tiwari, M. Rosen, and A. Madabhushi, "A hierarchical spectral clustering and nonlinear dimensionality reduction scheme for detection of prostate cancer from magnetic resonance spectroscopy (MRS)," Medical Physics, vol. 36, no. 9Part1, pp. 3927-3939, 2009.

[136] G. Litjens, O. Debats, J. Barentsz, N. Karssemeijer, and H. Huisman, "Computer-aided detection of prostate cancer in MRI," IEEE Transactions on Medical Imaging, vol. 33, no. 5, pp. 1083-1092, 2014.

[137] D. M. Saman, A. M. Lemieux, M. Nawal Lutfiyya, and M. S. Lipsky, "A review of the current epidemiology and treatment options for prostate cancer," Disease-a-Month, vol. 60, no. 4, pp. 150-154, 2014.

[138] M. Keyes, J Crook, G Morton, E Vigneault, N Usmani, and W. J Morris, "Treatment options for localized prostate cancer," Canadian family physician Medecin de famille canadien, vol. 59, no. 12, pp. 1269-1274, 2013.

[139] J. Yamamura, "MR imaging of prostate cancer: diffusion weighted imaging and (3D) hydrogen $1(1 \mathrm{H})$ MR spectroscopy in comparison with histology," 2011.

[140] A. Oto, C. Yang, A. Kayhan et al., "Diffusion-weighted and dynamic contrast-enhanced MRI of prostate cancer: correlation of quantitative MR parameters with Gleason score and tumor angiogenesis," American Journal of Roentgenology, vol. 197, no. 6, pp. 1382-1390, 2011.

[141] T. Kobus, T. Hambrock, C. A. Hulsbergen-van de Kaa et al., "In vivo assessment of prostate cancer aggressiveness using magnetic resonance spectroscopic imaging at $3 \mathrm{~T}$ with an endorectal coil," European Urology, vol. 60, no. 5, pp. 1074-1080, 2011.

[142] K. L. Zakian, K. Sircar, H. Hricak et al., "Correlation of proton MR spectroscopic imaging with gleason score based on step-section pathologic analysis after radical prostatectomy," Radiology, vol. 234, no. 3, pp. 804-814, 2005.

[143] J. J. A. van Asten, V. Cuijpers, C. Hulsbergen-van de Kaa et al., "High resolution magic angle spinning NMR spectroscopy for metabolic assessment of cancer presence and Gleason score in human prostate needle biopsies," Magnetic Resonance Materials in Physics, Biology and Medicine, vol. 21, no. 6, pp. 435-442, 2008.

[144] M. Garcia-Martin, "Quantitative $1 \mathrm{H}$ MR spectroscopic imaging of the prostate gland using LCModel and a dedicated basis-set: correlation with histologic findings," Magnetic Resonance in Medicine, vol. 65, no. 2, pp. 329-339, 2011.

[145] T. W. J. Scheenen, S. W. T. P. J. Heijmink, S. A. Roell et al., "Three-dimensional proton MR spectroscopy of human prostate at 3 T without endorectal coil: feasibility," Radiology, vol. 245 , no. 2, pp. 507-516, 2007.

[146] A. Shukla-Dave, H. Hricak, N. M. Ishill et al., "Correlation of MR imaging and MR spectroscopic imaging findings with $\mathrm{Ki}-67$, phospho-Akt, and androgen receptor expression in prostate cancer," Radiology, vol. 250, no. 3, pp. 803-812, 2009.

[147] D. H. Lee, K. C. Koo, S. H. Lee et al., "Tumor lesion diameter on diffusion weighted magnetic resonance imaging could help predict insignificant prostate cancer in patients eligible for active surveillance: preliminary analysis," Journal of Urology, vol. 190, no. 4, pp. 1213-1217, 2013.

[148] K. Cam, S. Yucel, L. Turkeri, and A. Akdas, "Accuracy of transrectal ultrasound guided prostate biopsy: histopathological correlation to matched prostatectomy specimens," International Journal of Urology, vol. 9, no. 5, pp. 257-260, 2002.

[149] P. Quann, D. F Jarrard, and W Huang, "Current prostate biopsy protocols cannot reliably identify patients for focal therapy: correlation of low-risk prostate cancer on biopsy with radical prostatectomy findings," International Journal of Clinical and Experimental Pathology, vol. 3, no. 4, pp. 401-7, 2010.

[150] B. Djavan, V. Ravery, A. Zlotta et al., "Prospective evaluation of prostate cancer detected on biopsies 1,2,3 and 4: when should we stop?" Journal of Urology, vol. 166, no. 5, pp. 1679-1683, 2001.

[151] P. Tiwari, A. Madabhushi, and M. Rosen, "A hierarchical unsupervised spectral clustering scheme for detection of prostate cancer from magnetic resonance spectroscopy (MRS)," in Proceedings of the International Conference on Medical Image Computing and Computer-Assisted Intervention, Springer, Brisbane, Australia, October 2007.

[152] S. Viswanath, "A comprehensive segmentation, registration, and cancer detection scheme on 3 Tesla in vivo prostate DCE-MRI," in Proceedings of the International conference on medical image computing and computer-assisted intervention, Springer, New York, NY, USA, September 2008.

[153] S. Viswanath, "Integrating structural and functional imaging for computer assisted detection of prostate cancer on multiprotocol in vivo 3 Tesla MRI," in Medical Imaging 2009: Computer-Aided DiagnosisInternational Society for Optics and Photonics, Bellingham, WA, USA, 2009.

[154] P. C. Vos, T. Hambrock, J. O. Barenstz, and H. J. Huisman, "Computer-assisted analysis of peripheral zone prostate lesions using T2-weighted and dynamic contrast enhanced T1weighted MRI," Physics in Medicine and Biology, vol. 55, no. 6, pp. 1719-1734, 2010.

[155] P. Liu, "A prostate cancer computer-aided diagnosis system using multimodal magnetic resonance imaging and targeted biopsy labels," in Medical Imaging 2013: Computer-Aided DiagnosisInternational Society for Optics and Photonics, Bellingham, WA, USA, 2013.

[156] M. Moradi, S. E. Salcudean, S. D. Chang et al., "Multiparametric MRI maps for detection and grading of dominant prostate tumors," Journal of Magnetic Resonance Imaging, vol. 35, no. 6, pp. 1403-1413, 2012.

[157] Y. Artan, M. A. Haider, D. L. Langer et al., "Prostate cancer localization with multispectral MRI using cost-sensitive support vector machines and conditional random fields," IEEE Transactions on Image Processing, vol. 19, no. 9, pp. 2444-2455, 2010.

[158] P. Tiwari, J. Kurhanewicz, and A. Madabhushi, "Multi-kernel graph embedding for detection, Gleason grading of prostate cancer via MRI/MRS," Medical Image Analysis, vol. 17, no. 2, pp. 219-235, 2013.

[159] P. Tiwari, S. Viswanath, J. Kurhanewicz, A. Sridhar, and A. Madabhushi, "Multimodal wavelet embedding representation for data combination (MaWERiC): integrating 
magnetic resonance imaging and spectroscopy for prostate cancer detection," NMR in Biomedicine, vol. 25, no. 4, pp. 607-619, 2012.

[160] J. F. G. Molina, "Incremental learning with SVM for multimodal classification of prostatic adenocarcinoma," PLoS One, vol. 9, no. 4, Article ID e93600, 2014.

[161] J. T. Kwak, S. Xu, B. J. Wood et al., "Automated prostate cancer detection usingT2-weighted and high-b-value diffusion-weighted magnetic resonance imaging," Medical Physics, vol. 42, no. 5, pp. 2368-2378, 2015.

[162] K. Zhao, C. Wang, J. Hu et al., "Prostate cancer identification: quantitative analysis of T2-weighted MR images based on a back propagation artificial neural network model," Science China Life Sciences, vol. 58, no. 7, pp. 666-673, 2015.

[163] Z. Wang, C. Liu, D. Cheng, L. Wang, X. Yang, and K.-T. Cheng, "Automated detection of clinically significant prostate cancer in mp-MRI images based on an end-to-end deep neural network," IEEE Transactions on Medical Imaging, vol. 37, no. 5, pp. 1127-1139, 2018.

[164] U. Sahrin, "Prostate cancer classification technique on pelvis CT images," International Journal of Engineering and Technology, vol. 8, no. 1.2, pp. 206-213, 2019.

[165] H. Xu, J. S. H. Baxter, O. Akin, and D. Cantor-Rivera, "Prostate cancer detection using residual networks," International Journal of Computer Assisted Radiology and Surgery, vol. 14, no. 10, pp. 1647-1650, 2019. 